\title{
Modelling the effect of the radiation reaction force on the acceleration of ultra-thin foils
}

\author{
M. J. Duff ${ }^{1}$, R. Capdessus ${ }^{1}$, M. King ${ }^{1}$, D. Del Sorbo ${ }^{2}$, C. P. Ridgers ${ }^{2}$ and P. McKenna ${ }^{1}$ \\ ${ }^{1}$ SUPA, Department of Physics, University of Strathclyde, Glasgow G4 0NG, UK \\ ${ }^{2}$ York Plasma Institute, Department of Physics, University of York, York YO10 5DQ, UK
}

\begin{abstract}
An investigation of the effects of the radiation reaction force on radiation pressure acceleration is presented. Through $1 \mathrm{D}(3 \mathrm{~V})$ PIC code simulations, it is found that radiation reaction causes a decrease in the target velocity during the interaction of an ultra-intense laser pulse with a solid density thin foil of varying thickness. This change in the target velocity can be related to the loss of backwards-directed electrons due to cooling and reflection in the laser field. The loss of this electron population changes the distribution of the emitted synchrotron radiation. We demonstrate that it is the emission of radiation which leads to the observed decrease in target velocity. Through a modification to the light sail equation of motion (which is used to describe radiation pressure acceleration in thin foils), which accounts for the conversion of laser energy to synchrotron radiation, we can describe this change in target velocity. This model can be tested in future experiments with ultra-high intensity lasers, and will lead to a better understanding of the process of relativistically induced transparency in the new intensity regime.
\end{abstract}

Keywords: High-field physics, radiation reaction force, radiation pressure acceleration

\section{INTRODUCTION}

The multi-petawatt (PW) laser facilities under development will enable experimental investigations into the interaction of ultra-intense laser pulses with plasma. Laser plasma interaction physics is an active area of research with many different aspects, including fast electron transport, ${ }^{1}$ ion acceleration, ${ }^{2,3}$ absorption physics ${ }^{4}$ and fundamental QED studies. ${ }^{5,6}$ Ion acceleration is a topic which will receive particular attention at multi-PW laser facilities due to its application to cancer therapies. ${ }^{7}$ At the intensities which will be achieved with multi-PW systems, high energy ions are predicted to be achieved through radiation pressure acceleration (RPA), where the radiation pressure due to the laser light is sufficiently high to accelerate thin foil targets to high velocities. RPA is expected to produce quasi-monoenergetic ion bunches, where the maximum energy has a fast scaling with the laser intensity. ${ }^{8,9}$ The maximum achievable ion energies may however be limited by QED effects, such as radiation reaction, which will become important in future facilities.

Radiation reaction (RR) primarily affects the electron dynamics, ${ }^{10,11}$ but it also indirectly impacts ions through modification of the self-consistent fields within the plasma. The effects of RR on ion acceleration is an active research area. Previous work ${ }^{12,13}$ has shown that the emission of synchrotron radiation causes a reduction in the laser piston velocity during the hole boring phase of RPA, leading to reduced ion energies. For thin targets however $\left(l<\lambda_{L}\right)$, there is an increase in ion energy due to enhancement of the charge separation fields within the plasma. These enhanced fields are the result of radiative cooling of the electrons which counter-propagate against the laser. These electrons are subsequently deflected by the laser, creating a dense electron bunch at the laser-plasma interface. This produces a strong charge separation field which subsequently accelerates ions.

The effects of RR on ion acceleration in the light sail regime of RPA have previously been investigated by Chen et al. ${ }^{14}$ This study suggests that it is the counter-propagating electron population which will experience the strongest RR effects, causing the electron phase space to contract. ${ }^{15}$ This can lead to enhanced ion acceleration (i.e. higher ion energy and conversion efficiency) for certain target and laser parameters. Tamburini $e t a l^{16}$ have

Further author information: (Send correspondence to M. J. Duff and P. McKenna)

M. J. Duff, E-mail: matthew.duff@strath.ac.uk, P. McKenna, E-mail: paul.mckenna@strath.ac.uk 
also investigated RR effects in the light sail regime, in particular noting that the effects are best seen in linear polarisation and again describing the formation of high density electron bunches due to radiative cooling.

Here, we present a further study into the effects of RR on the dynamics of solid density targets undergoing light sail-like acceleration. The structure of this paper is as follows; we begin with a discussion of some relevent physical concepts, including the production of synchrotron radiation, radiation reaction and the equation of motion of a radiating electron. We then briefly discuss the particle-in-cell approach to simulations of laser plasma interactions before presenting the preliminary results of a $1 \mathrm{D}(3 \mathrm{~V})$ simulation study into RR effects on target velocity. Finally, these results are compared to existing models for LS acceleration.

\section{THEORETICAL APPROACH}

\subsection{Electron dynamics in strong fields}

Electrons in ultra-intense laser fields oscillate at relativistic velocities. The threshold for relativistic electron motion is characterised by a parameter known as the normalised laser vector potential, $a_{0}$. When $a_{0}=1$, the work done by the laser over the period of a wavelength is equal to the rest mass of the electron. The $a_{0}$ parameter is defined as follows:

$$
a_{0}=\frac{e E}{m_{e} c \omega_{L}}
$$

In equation (1),e, $m_{e}$ are the electron charge and mass (respectively), $c$ is the speed of light, $E$ is the laser electric field strength and $\omega_{L}$ is the laser frequency. Electrons oscillating in ultra-intense laser fields are strong sources of synchrotron radiation. The photons of emitted radiation carry away momentum, therefore the electrons must be subject to a recoil force in order for their motion to conserve momentum. The equation of motion for radiating electrons therefore depends on the force from external fields (through the Lorentz force) as well as the recoil force exerted on the electrons through emission of synchrotron radiation. Although radiation reaction (RR) is fundamentally a quantum process, it is assumed that it can be treated using a classical approach, in which the recoil force introduces small corrections to the electron trajectory. ${ }^{17-19}$ This approach is valid provided that the RR force experienced by an electron in its rest frame is much weaker than the Lorentz force. The electron trajectory is then described by the Landau-Lifshitz equation: ${ }^{20}$

$$
m \ddot{x}^{\mu}=e F_{e x t}^{\mu \nu} \dot{x}_{\nu}+\tau_{r} e \partial_{\gamma} F_{e x t}^{\mu \nu} \dot{x_{\nu}} \dot{x}^{\gamma}+\tau_{r} \frac{e^{2}}{m} \triangle^{\mu}{ }_{\nu} F_{e x t}^{\nu \gamma} F_{\gamma \delta}^{e x t} \dot{x}^{\delta}
$$

Here, $x^{\mu}$ is the position 4 -vector, with dots representing differentiation with respect to proper time. $\tau_{r} \simeq$ $6.2 \times 10^{-24}$ is the classical radiation time, and $\triangle^{\mu}{ }_{\nu}=\delta^{\mu}{ }_{\nu}+\dot{x}^{\mu} \dot{x}_{\nu}$ is the orthogonal projection of the electron velocity. The indices extend over the space-time co-ordinates of the 4 -vectors present in the equation, i.e. $\mu, \nu, \gamma, \delta \in\{0,1,2,3\} . \mathrm{F}^{\mu \nu}$ is the electromagnetic tensor describing the external (laser) fields.

As the Landau-Lifshitz equation provides a classical description of radiation reaction, it does not account for quantum effects such as pair-production or non-linear scattering. The importance of these quantum effects can be quantified by a Lorentz invariant parameter, $\chi$ :

$$
\chi=\frac{\sqrt{F_{\nu}^{\mu} F_{\gamma}^{\nu} \dot{x}_{\mu} \dot{x}^{\gamma}}}{E_{S c h}}
$$

The $\chi$ parameter is the ratio of the electric field experienced by an electron in its rest frame to the Schwinger field, $\mathrm{E}_{S c h}=m_{e}^{2} c^{3} / e \hbar$. This Schwinger field is the upper limit on the intensity of an electromagnetic field, beyond which the vacuum breaks down to produce electron-positron pairs. ${ }^{21}$ When $\chi \simeq 1$, strong field effects such as pair production and non-linear scattering play an important role in the electron dynamics. However, signatures of RR for electrons with $\chi>0.1$ have been predicted by Blackburn et al, ${ }^{22}$ well before the classical model provided by the Landau-Lifshitz equation breaks down.

The classical approach described here overestimates the radiated power from an electron and therefore also overestimates the magnitude of the radiation reaction force. This is typically handled by scaling the radiated 
power by a function $\mathrm{g}(\chi)$, which reduces the contribution from electrons with high $\chi$ values. In the simulations presented here, $\chi \simeq 0.1$, which leads to a reduction in the radiated power of a factor $0.66{ }^{23}$ The overestimation of the radiated power is a result of the stochastic nature of photon emission which is not described in the classical approach. In the simulations however, the stochastic effects are accounted for by using a Monte-Carlo approach to calculate the probability of photon emission.

When the energy radiated by an electron in a laser plasma interaction is equal to its initial kinetic energy, the interaction enters the radiation dominated regime (RDR). ${ }^{24}$ Here, RR significantly affects the electron dynamics, since the emission of high energy photons causes discontinuities in the electron trajectory. It is difficult to enter the RDR due to current limitations in laser intensity, as described in reference. ${ }^{11}$ However, another regime in which the RR force plays an important role in the electron dynamics, and which is more easily acessible than the RDR, has been described by Di Piazza et al. ${ }^{11}$ In this regime, it is a population of electrons which counter propagates with respect to the laser which experiences the strong RR effects. In laser interactions with overdense solids, part of the laser pulse is reflected from the target in the initial stage of the interaction, before it undergoes relativistic self-induced transparency (RSIT). The reflected wave and incident laser pulse interfere, producing a standing wave in front of the target. As the surface is ionised and a plasma forms, the electrons in the plasma will interact with the standing wave set up at the target surface. Some of the electrons will counter propagate with respect to the laser and will therefore experience up-shifted laser fields. These electrons are stimulated to radiate away their energy and can subsequently be reflected by the laser, leading to the loss of this backwards moving population. The condition which the electrons must satisfy to be reflected by the laser is: ${ }^{11}$

$$
\frac{4 \gamma^{2}-a_{0}^{2}}{2 a_{0}^{2}}>0
$$

It is worth emphasizing that this reflection can only occur when RR effects are included in the electron dynamics, since equation (4) describes the loss of momentum from radiating electrons. The electrons which are reflected by the laser become trapped in the plasma, where they can enhance the absorption of laser energy. This bunch of trapped electrons also leads to the formation of an enhanced charge separation field, as has been described by Chen et al. ${ }^{14}$ This backwards propagating population of electrons can also be produced as the target undergoes RSIT. In this case, the laser propagates into the target and heats the electrons within the target volume. The plasma will then expand outwards, with some fraction of the plasma electrons counter propagating with respect to the laser and therefore being reflected as described in equation (4).

\subsection{Synchrotron radiation}

In ultra-intense laser plasma interactions, the emission of synchrotron radiation originates from the acceleration of electrons in the both the laser fields and the self consistent fields within the plasma. As discussed in the previous subsection, the emission of photons of synchrotron radiation cause a recoil force to act on a radiating electron, which must be accounted for in the equation of motion. Here we will briefly discuss the properties of the emitted radiation.

The generation of synchrotron radiation arises as a consequence of the finite speed of light. Many of the subtleties involved in the analysis of the emitted radiation arise due to the fact the radiation seen by an observer from an accelerating charge has originated from a time earlier in the particle's motion, when it was at a different location. The electromagnetic fields associated with the charge are obtained from the Lienard-Wiechert potentials, such that the fields contain two components. The first is a static field, which is seen by the electron in its rest frame and measured by an observer co-moving with respect to the charge. The second field component depends on the retarded time and describes the radiated field originating from the accelerated charge. Substituting these fields into the Poynting vector yields an expression for the intensity of emitted radiation, as described in references. ${ }^{25,26}$

The spectrum of synchrotron radiation generated from electrons oscillating in laser fields consists of many Doppler shifted harmonics of the laser frequency. These harmonics extend up to a critical frequency at which the spectrum peaks. The critical frequency is related to the energy of the radiating electron and the frequency of its rotation in the laser field, $\omega_{r}$ by the expression $\omega_{c r} \simeq \frac{3}{2} \omega_{r} \gamma_{e}^{3}$. 
In the case of ultra-relativistic electrons, the synchrotron radiation is emitted into a narrow cone along the direction of electron momentum, with an opening angle of $1 / \gamma_{e}$. It can therefore be assumed that the photons radiated by the ultra-relativistic electron also travel in the same direction, since the opening angle will be small. The spectral intensity of the synchrotron radiation per unit solid angle is given by: ${ }^{19}$

$$
\frac{d^{2} I}{d \omega \Omega}=\frac{\gamma_{e}^{3} \tau_{r}}{\omega_{c r}}\left(\mathbf{F}_{L e} \cdot \ddot{\mathbf{x}}_{e}\right) \delta\left(\Omega-\frac{\mathbf{p}_{e}}{p_{e}}\right) S\left(\frac{\omega}{\omega_{c r}}\right)
$$

The delta function ensures that the radiation is emitted only into a small solid angle along the direction of electron momentum. Here, $\ddot{\mathbf{x}}_{e}$ is the acceleration of the electron, $\mathbf{p}_{e}$ is the electron momentum and $\mathbf{S}$ is a function (known as the universal function of synchrotron radiation) which defines the shape of the spectrum.

As discussed, synchrotron radiation is only one specific example of the radiation produced by accelerating charges. Bremsstrahlung radiation is a more generic term applied to the emission from charges which are decelerated as they propagate through matter. The radiated synchrotron power roughly scales with $a_{0}^{4}$, meaning that in ultra-relativistic laser pulses with $a_{0}>100$ that the synchrotron emission will dominate over Bremsstrahlung. ${ }^{20}$ This has important consequences for experimental tests of RR, where separating the synchrotron signal from other radiation sources will be a key objective. It is especially important to obtain a clear synchrotron spectrum in order to distinguish quantum RR effects, which will be an objective of multi-PW experiments.

\subsection{Radiation pressure acceleration}

During ultra-intense laser plasma interactions, the radiation pressure exerted on the target as a result of the high incident photon flux, can cause it to accelerate to high velocities. This is known as radiation pressure acceleration (RPA). Ultra thin targets (such as nanometer scale foils) undergo acceleration in the light sail (LS) regime. In this case the laser directly accelerates the target electrons, establishing a charge separation field which subsequently accelerates ions to high energies. When viewed over a sufficiently long timescale, the dynamics of such a system can be modeled by a co-moving layer of electrons and ions, 'sailing' under the influence of the laser.

The LS regime is highly desirable for ion acceleration experiments, due to the high energy ions which can be produced, in quasi-monoenergetic bunches. Additionally, the ion energy scales with the square of the laser intensity (albeit in the non-relativistic regime), much faster than other ion acceleration regimes such as target normal sheath acceleration (TNSA). The idea of the LS was originally proposed by Marx, ${ }^{27}$ who suggested that a laser could be used to propel a spacecraft over interstellar distances. A subsequent review of the original LS equations and the feasibility of such a method of travel can be found in reference. ${ }^{28}$

The equation of motion for an ultra-thin foil undergoing RPA under the influence of an intense laser pulse was later revisited by Macchi et al, ${ }^{29}$ by accounting for the effects of the charge separation field and RSIT. The equation can be derived by considering the target to act as a perfectly reflecting mirror, gaining momentum as the laser photons hit the target surface. As the target accelerates to a significant fraction of the speed of light, the laser intensity arriving at the target surface (as measured in the rest frame) is reduced by the Doppler effect. The intensity in the rest frame is therefore related to the laser intensity in the laboratory frame by a Doppler shift; $I^{\prime}=I(1-\beta) /(1+\beta)$. The radiation pressure exerted on the target is:

$$
P_{\text {rad }}=\frac{I^{\prime}}{c}(1+R-T)=\frac{I^{\prime}}{c}(2 R+A)
$$

Here, R, T and A represent the reflection, transmission and absorption coefficients, respectively, such that $\mathrm{R}+\mathrm{T}+\mathrm{A}=1$. If the target undergoes RSIT at some point in the interaction, the high transmission of laser light will suddenly reduce the radiation pressure arriving at the target surface, as there is no longer a solid surface to push against.

To arrive at the equation presented in reference, ${ }^{29}$ we assume that there is no absorption, and consider the reflection coefficient as measured in the target frame of reference. This reflection then depends on the frequency in the target rest frame, $\omega^{\prime}$, which is related to the frequency in the laboratory frame by: $\omega^{\prime}=\omega \sqrt{(1-\beta) /(1+\beta)}$. These assumptions lead to the LS equation of motion: 


$$
\frac{d}{d t}(\beta \gamma)=\frac{2 I}{\rho l c^{2}} R\left(\omega^{\prime}\right) \frac{1-\beta}{1+\beta}
$$

For the simple case of a perfectly reflecting mirror, where $\mathrm{R}=1$, the velocity of the target is found by integrating the equation of motion with respect to the retarted time $\phi=t(1-\beta)$ (using the approach described by Simmons and McInnes ${ }^{28}$ ) to obtain:

$$
\beta=\frac{(1+\xi)^{2}-1}{(1+\xi)^{2}+1} \quad ; \quad \xi=\frac{2 F}{\rho l c^{2}} \quad \text { where } \quad F=\int_{0}^{\phi} I\left(\phi^{\prime}\right) d \phi^{\prime}
$$

Through the parameter $\xi$, the LS velocity depends on the laser fluence as well as target properties such as density and thickness. Moving to thinner and less dense targets suggests that the LS velocity will increase. There is however a limit to this approach; if the targets are sufficiently thin they will undergo RSIT. The laser can then propagate through the target, switching off the radiation pressure acceleration since the target can no longer be accelerated as a whole. The threshold for transparency is when the $a_{0}$ of the laser exceeds the areal density of the target $\left(a_{0}>\zeta=\pi \frac{n_{e}}{n_{c}} \frac{l}{\lambda}\right)$.

In reality the reflectivity is less than 1, due to absorption of the incident laser energy and subsequent RSIT of the target which reduces the effectiveness of the RPA. In order to account for this effect, Macchi et al reformulated the equation of motion to include a non-linear reflection coefficient, which contains the areal density parameter. ${ }^{30}$ The reflection can be approximated by the following expressions:

$$
R=\left\{\begin{array}{llc}
1 /\left(1+\zeta^{-2}\right) & \text { when } & a_{0}<\sqrt{1+\zeta^{2}} \\
\frac{\zeta^{2}}{a_{0}^{2}} & \text { when } & a_{0}>\sqrt{1+\zeta^{2}}
\end{array}\right.
$$

For target thicknesses in which the areal density exceeds the laser $a_{0}$, the reflection becomes independent of the laser intensity. This suggests that RPA reaches peak efficiency when the laser $a_{0}$ is equal to the target thickness. This condition also defines the threshold for RSIT.

Some aspects of the effects of RR in the light sail regime have been investigated by Chen et al ${ }^{14}$ and Tamburini et al. ${ }^{16}$ Chen concludes that RR effects are most signifiant in the population of electrons which counter-propagate with respect to the laser pulse. This leads to a boost in the ion energy in thin targets. Tamburini reports that the RR effects are important for linear polarisation, and that the ion conversion efficiency with RR on is lower due to a decrease in the return current.

\section{SIMULATION RESULTS AND DISCUSSION}

\subsection{Simulation Parameters}

Here we present the results of $1 \mathrm{D}(3 \mathrm{~V})$ simulations using the EPOCH Particle-In-Cell code. ${ }^{31} 1 \mathrm{D}$ simulations have been used in order to scan over a wide range of parameters with sufficiently high resolution. We have investigated the interaction of solid density $\left(777 n_{c}\right)$ aluminium targets, in a charge state of $13^{+}$, with an ultra-intense laser pulse of intensity $2 \times 10^{23} \mathrm{Wcm}^{-2}$. At this intensity, the RR force plays an important role in the electron dynamics whilst higher order QED effects such as pair-production can be neglected. The targets are varied in thickness from $50-500 \mathrm{~nm}$, such that during the interaction they are accelerated in the light sail like-regime of RPA.

The simulations have a spatial resolution of $2 \mathrm{~nm}(100,000$ cells across a grid size of $200 \mu \mathrm{m})$, in order to fully resolve the Debye length and avoid artificial heating. Dynamic load balancing is used to ensure a high number of particles per cell $(>1000)$ throughout the simulations.

The laser has a Gaussian temporal profile, with a FWHM of $60 \mathrm{fs}$ and a peak dimensionless laser amplitude of $a_{0}=380$. The wavelength is $1 \mu m$, with outputs produced every laser period $T_{L}=2 \pi / \omega_{L}$. The results presented here are for linearly polarised laser pulses (LP), as this choice of polarisation was found to enhance RR effects due to increased electron heating compared to the circularly polarised case. ${ }^{16}$ 

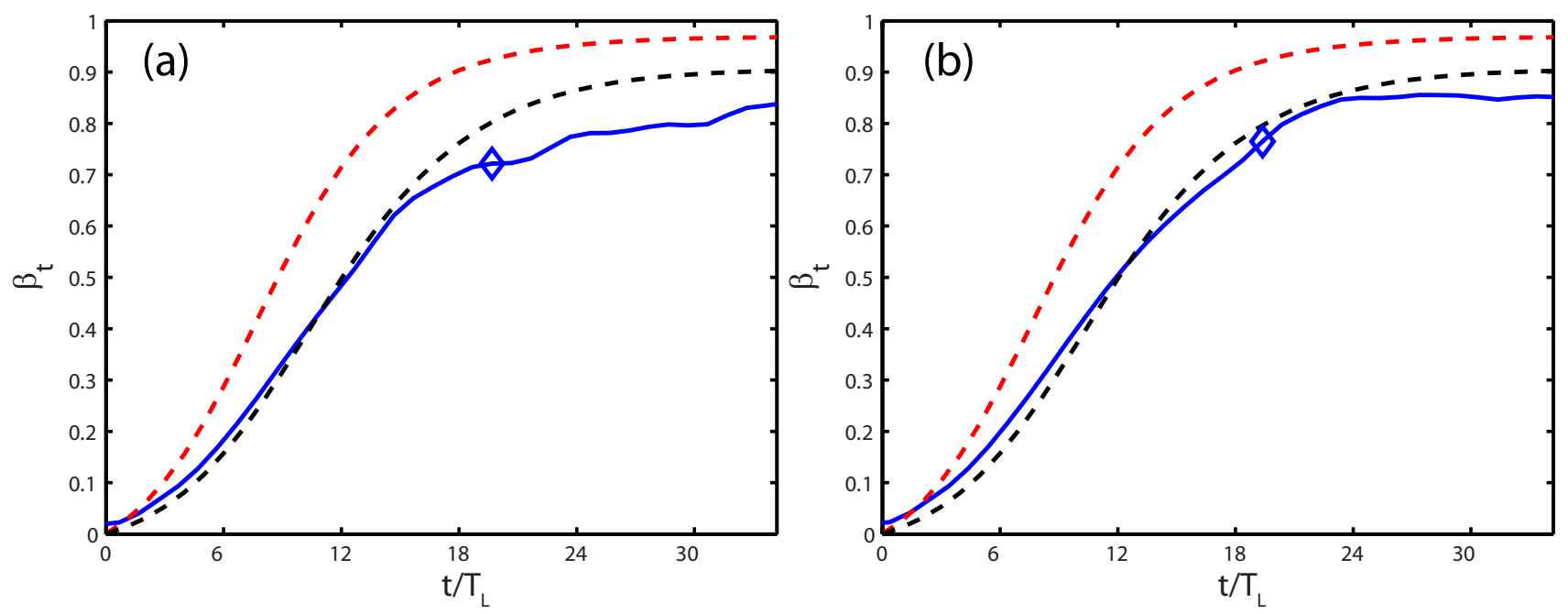

Figure 1. Normalised target velocity as a function of time for the $150 \mathrm{~nm}$ simulation, with RR on (a) and off (b). The solid blue line shows the target velocity obtained from 1D simulations. The dashed red and black lines denote the target velocity calculated with the Macchi model, assuming $R=1$ (red dashed line) and a non-linear reflection coefficient (dashed black line). The blue diamond in each plot marks the time of maximum synchrotron emission, the time at which the velocities are compared in Figure 2.

The RR force is implemented using the QED package in EPOCH. This package uses a semi-classical approach, in which the photon emission from a radiating electron is handled stochastically ${ }^{18,32}$. The momentum carried away by the photon then causes the radiating electron to recoil, changing its trajectory. In order to isolate effects which arise purely due to the RR force, the simulations were run with and without RR. With RR switched off, the electrons still produce synchrotron radiation, however the recoil of the emitted photon is not accounted for.

\subsection{Target Dynamics}

At the high intensities presented in this study, the target undergoes RPA in a regime similar to LS. We here refer to a LS-like regime, because some assumptions used in the derivation of the previously presented LS model do not hold, such as perfect reflectivity of the target and negligible absorption of the laser pulse. Additionally, the $\mathrm{RR}$ force changes the dynamics of the system, meaning a different model is needed to describe the motion of the target.

Figure 1 shows the target velocity as a function of time for the $150 \mathrm{~nm}$ case, with and without $\mathrm{RR}^{1}$. The 150 $\mathrm{nm}$ case was chosen here because this is the target thickness at which the laser $a_{0}$ approximately balances the target areal density, which leads to the most efficient RPA as described in reference. ${ }^{30}$

Figure 1 clearly shows that the targets are undergoing RPA similar to that of the LS regime, since the velocity is of the order predicted by the LS model. The solid blue line marks the target velocity measured from simulations as a function of time. This velocity is compared to the Macchi LS model with various assumptions. The dashed red line indicates the Macchi LS velocity, assuming that the target acts like a perfectly reflecting mirror $(R=1) .{ }^{29}$ Different pulse shapes can be accounted for in this model through an appropriate choice of laser fluence; in Figure 1 the fluence has been modified to account for the Gaussian temporal profile of the laser. From Figure 1 we can see that this model overestimates the target velocity compared to the simulation. This is to be expected; in reality the target will absorb some fraction of the laser energy and for the thickness considered, will go transparent due to RSIT. However, the model and simulation results exhibit the same trend. The target velocity increases up until the maximum value of the laser fluence, at which point it reaches a constant velocity. If the target was being accelerated in the hole-boring regime of RPA, the velocity would peak at with the laser

\footnotetext{
${ }^{1}$ The target velocity is determined by tracking the position of the maximum electron density surface throughout the simulation.
} 


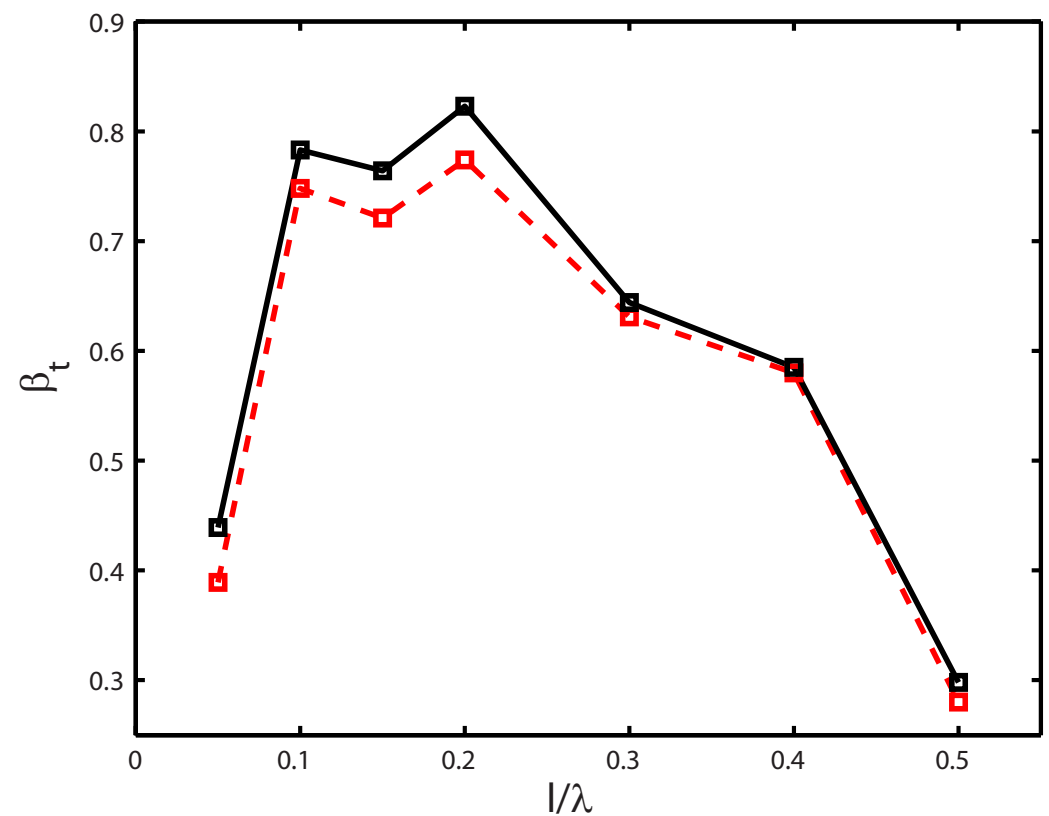

Figure 2. Normalised target velocity as a function of target thickness, with and without RR (red dashed line and black solid line respectively) and measured at the time of maximum synchrotron emission. With RR effects included, the target velocity decreases.

intensity (at $60 \mathrm{fs}$ ) and subsequently decrease. The velocity would also be much lower, peaking at approximately $\beta_{t}=0.1$ for a $150 \mathrm{~nm}$ target.

For the $150 \mathrm{~nm}$ case presented in Figure 1, the laser $a_{0}$ marginally exceeds the target areal density, $\zeta=\pi \frac{n_{e}}{n_{c}} \frac{l}{\lambda}$. In this case, reference ${ }^{33}$ provides an estimate of the non-linear reflection coefficient, which depends on the laser and target parameters. The non-linear reflectivity accounts for the fact that the target can undergo RSIT during the interaction. The target velocity calculated using this non-linear reflection coefficient is plotted in Figure 1 with a black dotted line. Using this estimate of the reflection coefficient, there is better agreement with the simulation results.

The target velocity is compared with and without RR in Figure 1. When RR effects are included, the target velocity is seen to decrease, falling below the velocity predicted by the Macchi model with non-linear reflection. The lack of agreement with the model in this case suggests that the RR force causes a change in the target velocity which has not previously been accounted for. Note here that the change in velocity is relatively small (around 5\%), so this difference could be accounted for by considering the RR force as a small perturbation to the LS equation of motion. The decrease in velocity is therefore related to the emission of radiation; in order to compare this effect, the LS velocity is calculated at the time of maximum synchrotron emission, at which point the largest differences will be observed. The blue diamond plotted in Figure 1 marks the time of maximum synchrotron emission, determined by the case with RR on.

The target velocity is presented as a function of target thickness in Figure 2. There are distinct regions in this plot, in which different effects are taking place. The Macchi model predicts that the optimal target thickness for RPA is obtained when the laser $a_{0}$ matches the areal density. For thicker targets, the reflection coefficient tends to 1 , meaning that the momentum transfer from laser photons to the target is as efficient as possible. However as the targets become thicker, their inertia prevents efficient acceleration, meaning that the optimal thickness occurs for the thinnest target in which the reflection coefficient is approximately 1, i.e. when $a_{0} \simeq \zeta$.

For the simulation parameters used here, the optimal target thickness is calculated to be $l_{\text {opt }}=156 \mathrm{~nm}$. This calculation assumes that the laser intensity is constant (i.e. a top hat pulse), whereas here we use a laser pulse with a Gaussian temporal profile. The optimal thickness may then be shifted, as the target accelerates to high 
velocity in the rising edge of the laser pulse, resulting in a lower intensity reaching the front surface due to the Doppler effect. The targets with thickness less than the optimal value will undergo RSIT early in the interaction, reducing the efficiency of RPA as this requires the target to remain overdense.

For targets which are thicker than the optimal value, Macchi predicts that the radiation pressure becomes independent of the laser $a_{0} \cdot{ }^{30}$ In this case, RPA reaches its maximum efficiency and so increasing the target thickness means the velocity will decrease due to the target inertia preventing it from accelerating efficiently.

As discussed earlier, electrons oscillating in the laser fields will emit synchrotron radiation into a cone along the direction of the electron velocity, with a small opening angle of $\simeq 1 / \gamma_{e}$. Whilst the electrons radiate only into a narrow cone, the system contains many electrons moving in different directions, so it would be expected that some radiation would exist in all directions. When RR effects are included however, the counter propagating electron population is lost due to reflection by the laser. This means the average emission direction will shift and will instead be directed forwards along the laser axis since this is the direction in which most electrons move.

With RR, we found that the average emission angle decreases relative to the case without RR. Again, we emphasize that this is due to the loss of the counter propagating electrons. The smaller emission angle means that the radiation is primarily emitted in the forwards direction. Since the photons of synchrotron radiation are carrying away momentum, there will be a recoil force directed opposite to the average emission direction. As the average emission angle drops when $\mathrm{RR}$ is accounted for, the component of the RR force which acts along the direction of target motion will be larger, causing the target velocity to drop relative to the case without RR.

\subsection{Discussion}

We have presented a short overview of our research into the effects of RR on overdense targets in the LS-like regime. Previous work in this area has highlighted the importance of backwards propagating electrons on the dynamics of the system. Here, we report how the loss of this electron population through radiative cooling and reflection leads to a decrease in the target velocity. By comparing the target velocity as measured in $1 \mathrm{D}(3 \mathrm{~V})$ simulations to the predictions of the existing LS model, we have shown that RR leads to a reduction in the velocity which has not been accounted for.

\section{ACKNOWLEDGEMENTS}

This work was funded by the UK EPSRC (grant numbers EP/M018091/1, EP/P007082/1 and EP/M018156/1). We also acknowledge the use of the EPOCH PIC code and the EPSRC funded ARCHIE-WeSt supercomputer in the running of the simulations presented. Data associated with the research presented in this paper is accessible at http://dx.doi.org/10.15129/e7fda3c4-a5a4-47b4-a224-5d9b0aeaeb71.

\section{REFERENCES}

1. A. Bell and R. Kingham, "Resistive collimation of electron beams in laser-produced plasmas," Physical review letters $\mathbf{9 1}(3)$, p. 035003, 2003.

2. R. Snavely, M. Key, S. Hatchett, T. Cowan, M. Roth, T. Phillips, M. Stoyer, E. Henry, T. Sangster, M. Singh, et al., "Intense high-energy proton beams from petawatt-laser irradiation of solids," Physical Review Letters 85(14), p. 2945, 2000.

3. A. Robinson, P. Gibbon, M. Zepf, S. Kar, R. Evans, and C. Bellei, "Relativistically correct hole-boring and ion acceleration by circularly polarized laser pulses," Plasma Physics and Controlled Fusion 51(2), p. 024004, 2009.

4. S. Wilks, W. Kruer, M. Tabak, and A. Langdon, "Absorption of ultra-intense laser pulses," Physical review letters 69(9), p. 1383, 1992.

5. P. A. Dirac, "Classical theory of radiating electrons," Proceedings of the Royal Society of London. Series A, Mathematical and Physical Sciences, pp. 148-169, 1938.

6. A. Ilderton and G. Torgrimsson, "Radiation reaction in strong field qed," Physics Letters B 725(4), pp. 481486, 2013. 
7. J. Caron, J.-L. Feugeas, B. Dubroca, G. Kantor, C. Dejean, G. Birindelli, T. Pichard, P. Nicolaï, E. d'Humières, M. Frank, et al., "Deterministic model for the transport of energetic particles: Application in the electron radiotherapy," Physica Medica 31(8), pp. 912-921, 2015.

8. B. Qiao, M. Zepf, M. Borghesi, and M. Geissler, "Stable gev ion-beam acceleration from thin foils by circularly polarized laser pulses," Phys. Rev. Lett. 102, p. 145002, Apr 2009.

9. S. Palaniyappan, C. Huang, D. C. Gautier, C. E. Hamilton, M. A. Santiago, C. Kreuzer, A. B. Sefkow, R. C. Shah, and J. C. Fernández, "Efficient quasi-monoenergetic ion beams from laser-driven relativistic plasmas," Nature communications 6, 2015.

10. A. Zhidkov, J. Koga, A. Sasaki, and M. Uesaka, "Radiation damping effects on the interaction of ultraintense laser pulses with an overdense plasma," Phys. Rev. Lett. 88, p. 185002, Apr 2002.

11. A. Di Piazza, K. Hatsagortsyan, and C. Keitel, "Strong signatures of radiation reaction below the radiationdominated regime," Physical review letters 102(25), p. 254802, 2009.

12. R. Capdessus and P. McKenna, "Influence of radiation reaction force on ultraintense laser-driven ion acceleration," Physical Review E 91(5), p. 053105, 2015.

13. E. Nerush and I. Y. Kostyukov, "Laser-driven hole boring and gamma-ray emission in high-density plasmas," Plasma Physics and Controlled Fusion 57(3), p. 035007, 2015.

14. M. Chen, A. Pukhov, T.-P. Yu, and Z.-M. Sheng, "Radiation reaction effects on ion acceleration in laser foil interaction," Plasma Physics and Controlled Fusion 53(1), p. 014004, 2010.

15. T. Esirkepov, M. Borghesi, S. Bulanov, G. Mourou, and T. Tajima, "Highly efficient relativistic-ion generation in the laser-piston regime," Physical review letters 92(17), p. 175003, 2004.

16. M. Tamburini, F. Pegoraro, A. Di Piazza, C. H. Keitel, and A. Macchi, "Radiation reaction effects on radiation pressure acceleration," New Journal of Physics 12(12), p. 123005, 2010.

17. J. G. Kirk, A. Bell, and I. Arka, "Pair production in counter-propagating laser beams," Plasma Physics and Controlled Fusion 51(8), p. 085008, 2009.

18. C. Ridgers, J. G. Kirk, R. Duclous, T. Blackburn, C. Brady, K. Bennett, T. Arber, and A. Bell, "Modelling gamma-ray photon emission and pair production in high-intensity laser-matter interactions," Journal of Computational Physics 260, pp. 273-285, 2014.

19. R. Capdessus, E. d'Humières, and V. Tikhonchuk, "Modelling of radiation losses for ion acceleration at ultra-high laser intensities," in EPJ Web of Conferences, 59, p. 17019, EDP Sciences, 2013.

20. L. D. Landau and E. M. Lifshitz, "The classical theory of fields: Volume 2 (course of theoretical physics series)," 2000.

21. A. R. Bell and J. G. Kirk, "Possibility of prolific pair production with high-power lasers," Phys. Rev. Lett. 101, p. 200403, Nov 2008.

22. T. Blackburn, C. Ridgers, J. G. Kirk, and A. Bell, "Quantum radiation reaction in laser-electron-beam collisions," Physical review letters 112(1), p. 015001, 2014.

23. A. G. R. Thomas, C. P. Ridgers, S. S. Bulanov, B. J. Griffin, and S. P. D. Mangles, "Strong radiationdamping effects in a gamma-ray source generated by the interaction of a high-intensity laser with a wakefieldaccelerated electron beam," Phys. Rev. X 2, p. 041004, Oct 2012.

24. S. Bulanov, T. Z. Esirkepov, J. Koga, and T. Tajima, "Interaction of electromagnetic waves with plasma in the radiation-dominated regime," Plasma Physics Reports 30(3), pp. 196-213, 2004.

25. H. Wiedemann, "Synchrotron radiation," in Particle Accelerator Physics, pp. 300-336, Springer, 2003.

26. J. D. Jackson, Electrodynamics, Wiley Online Library, 1975.

27. G. Marx, "Interstellar vehicle propelled by terrestrial laser beam," Nature 211, 1966.

28. J. Simmons and C. McInnes, "Was marx right? or how efficient are laser driven interstellar spacecraft?," American journal of physics 61(3), pp. 205-207, 1993.

29. A. Macchi, S. Veghini, and F. Pegoraro, "light sail acceleration reexamined," Physical review letters 103(8), p. $085003,2009$.

30. A. Macchi, S. Veghini, T. V. Liseykina, and F. Pegoraro, "Radiation pressure acceleration of ultrathin foils," New Journal of Physics 12(4), p. 045013, 2010. 
31. T. Arber, K. Bennett, C. Brady, A. Lawrence-Douglas, M. Ramsay, N. Sircombe, P. Gillies, R. Evans, H. Schmitz, A. Bell, et al., "Contemporary particle-in-cell approach to laser-plasma modelling," Plasma Physics and Controlled Fusion 57(11), p. 113001, 2015.

32. A. Thomas, C. Ridgers, S. Bulanov, B. Griffin, and S. Mangles, "Numerical calculations of a high brilliance synchrotron source and on issues with characterizing strong radiation damping effects in non-linear thomson/compton backscattering experiments," arXiv preprint arXiv:1204.5259, 2012.

33. A. Macchi, "Theory of light sail acceleration by intense lasers: an overview," High Power Laser Science and Engineering 2, p. e10, 2014. 